\title{
Performance of Directional Collision Avoidance in Ad Hoc Networks
}

\author{
Yu Wang and Jose Joaquin Garcia-Luna-Aceves \\ Department of Computer Engineering \\ University of California at Santa Cruz \\ Santa Cruz, CA 95064, U.S.A. \\ $\{$ ywang, jj\}@cse.ucsc.edu
}

\begin{abstract}
This paper analyzes the performance of directional collision avoidance schemes, in which antenna systems are used to direct the transmission and reception of control and data packets in channel access protocols based on four-way collision avoidance handshake. We present an analytical model that considers both directional reception and the possible difference in gain between omni-directional and directional transmissions. Analytical results show that, when the directional collision avoidance scheme in which all transmissions are directional is augmented with directional receiving, one-hop throughput does not decrease due to the increased spatial reuse, even when the number of competing nodes within a region increases as long as the directional transmission/reception beamwidth is narrow. It is also shown that, as expected, the performance of directional collision avoidance schemes degrades when directional transmissions have much higher gain than omni-directional transmissions. However, this degradation is relatively small. Simulations of the IEEE 802.11 protocol and its directional variants validate the results predicted in the analysis; and show that side lobes affect little on throughput if they are reasonably low and the carrier sensing threshold is raised to make nodes less sensitive to channel activities. It is argued that the all-directional scheme in which both transmission and reception of packets is directional is the most attractive collision avoidance approach.
\end{abstract}

\section{Introduction}

Collision avoidance is very important in ad hoc networks to combat the "hidden terminal" problem that can degrade throughput significantly. The usual four-way collision avoidance handshake (RTS-CTS-data-ACK), as deployed in the popular IEEE 802.11 MAC protocol [1] and some other protocols, performs much better than the simple carrier sense multiple access (CSMA) protocols in ad hoc networks. However, its performance still degrades rapidly when the number of nodes competing for a shared channel increases moderately due to the much reduced spatial reuse [2]. Recently several MAC schemes that use directional antennas have been proposed to enhance the performance of existing omnidirectional collision-avoidance schemes $[3,4,5,6,7]$, which we call directional collision

\footnotetext{
* This work was supported in part by the Defense Advanced Research Projects Agency (DARPA) under Grant No. DAAD19-01-C-0026 and by the US Air Force/OSR under Grant No. F4962000-1-0330.
} 
avoidance protocols. However, the majority of the performance analyses of directional collision avoidance schemes have been done via simulations $[3,4,5,6,7]$, and there is little prior work on the analytical modeling of directional collision avoidance protocols. Wang and Garcia-Luna-Aceves [8] extended the model by Takagi and Kleinrock [9] to analyze three directional collision-avoidance schemes based on omni-directional packet reception, together with omni-directional and directional transmissions. The limitation of that work is that it assumes that the gain for omni-directional transmissions is the same as that for directional transmissions, and that all packet reception is omni-directional. In current systems, it is possible to have directional packet reception, and the range of directional transmissions can be longer than the range of omni-directional transmissions. Such limitation motivates our work reported in this paper and the rest of the paper is organized as follows.

Section 2 outlines the directional collision avoidance schemes that we study analytically and by simulation. Section 3 presents the first analytical study of directional collision avoidance in ad hoc networks that considers (a) the effect of directional transmitting and receiving on spatial reuse and collision avoidance, and (b) the effect of the differences in gains between omni-directional and directional transmissions. Our model generalizes the analytical model adopted in [8]. Section 4 presents the results of simulations carried out to validate the results from the analytical model, as well as to analyze the effect of side lobes in directional transmissions. The IEEE 802.11 MAC protocol and its variants that implement directional collision avoidance are investigated. Section 5 summarizes our results and outlines directions for future work.

\section{Directional Collision Avoidance Schemes}

In omni-directional MAC schemes, all packet transmissions and receptions are omnidirectional. The IEEE 802.11 MAC protocol is an example of a protocol based on this scheme. We call this scheme OTOR (for "omni-directional transmission and omnidirectional reception").

We consider two directional collision-avoidance schemes in our analytical study. In both schemes, RTS, CTS, data packets and ACK are transmitted directionally. When a node is transmitting in one direction, it appears "deaf" to other directions and cannot sense any channel activity at all.

One scheme uses omni-directional receiving mode whenever it is not transmitting; we call such a scheme DTOR (for "directional transmission and omni-directional reception"). In the other scheme, which we call DTDR (for "directional transmission and directional reception"), a node directs its antenna to the neighbor from which it expects to receive a packet. A node that is not expecting a packet from a neighbor listens to the channel omni-directionally.

It is also possible to establish schemes that combine both omni-directional and directional transmissions and receptions. For example, in one scheme, only CTS packets are transmitted omni-directionally, while all the other types of packets are transmitted directionally. Omni-directional or directional reception can be applied to this approach. However, directional reception is intuitively more attractive, and results in the MTDR scheme (for "mixed-directional transmission and directional reception"). 
To implement any of the directional collision avoidance schemes, nodes have to know the relative locations of their neighbors. In this paper, we assume that broadcast beacons are used by nodes to determine who their neighbors are and their relative locations. These broadcast beacons are transmitted omni-diretionally in the same channel as other packets and nodes communicate directly only with nodes within its omni-directional transmission range to avoid the complexity of maintaining additional neighbors that are only reachable through directional transmissions.

\section{Approximate Analysis}

In this section, we present the analysis of the DTOR and DTDR schemes and compare their performance with the existing omni-directional OTOR scheme. The MTDR scheme can be analyzed using the approach presented here, but its analysis is omitted for brevity. However, it is still compared with the other schemes later in simulations.

\subsection{Assumptions}

For simplicity, we assume that directional transmissions and receptions have equal beamwidth. Most of the assumptions made in our analysis of directional collision avoidance schemes have been used successfully in the performance evaluation of several MAC protocols $[10,9,11,2,8]$ to obtain tractable analytical models. Below we just highlight the key assumptions.

We assume a multi-hop network in which nodes are two dimensionally Poisson distributed with density $\lambda$, i.e., the probability $p(i, S)$ of finding $i$ nodes in an area of $S$ is given by:

$$
p(i, S)=\frac{(\lambda S)^{i}}{i !} e^{-\lambda S} .
$$

Each node has omni-directional and directional transmission ranges of $R$ and $R^{\prime}$ respectively. We have $R^{\prime}=\gamma R$, where $\gamma \geq 1$. Suppose $N$ is the average number of nodes within a circular region of radius $R$; therefore, we have $N=\lambda \pi R^{2}$. Similarly we have $N^{\prime}=\lambda \pi R^{\prime 2}=\gamma^{2} N$.

We use a time-slotted system as an approximation in which each time slot lasts $\tau$ and the transmission times of RTS, CTS, data, and ACK packets are normalized with regard to $\tau$ and are denoted by $l_{r t s}, l_{c t s}, l_{d a t a}$, and $l_{a c k}$, respectively.

We assume that a silent node begins transmission with probability $p$ at each time slot which is a protocol-specific parameter but is slot independent. As advocated in [8], we use a three-state Markov chain to derive the throughput which is largely decided by $p$.

The node model is shown in Fig. 1(from Fig. 1 in [8]), where wait is the state when the node defers for other nodes or backs off, succeed is the state when the node can complete a successful four-way handshake with other nodes, and fail is the state when the node initiates a handshake that is unsuccessful or cannot be completed due to collisions.

In our analytical modeling, we assume complete signal attenuation outside the main transmission beamwidth. In reality, beamforming antennas can generate side lobes whose interference range can be modeled by a distance parameter $d$. However, we reason that 


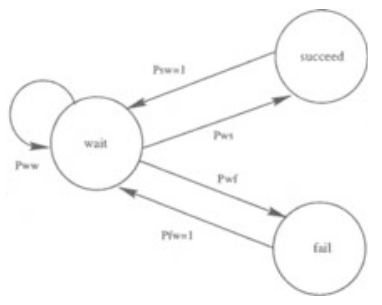

Fig. 1. Markov chain model for a node

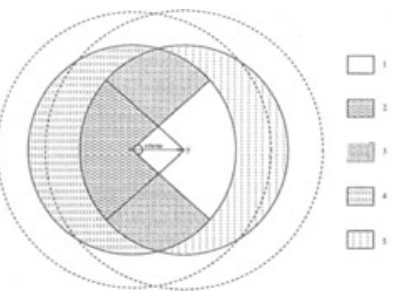

Fig. 2. The DTOR scheme

the influence of side lobes on throughput is insignificant if the side lobe level is low enough and the carrier sensing (CS) threshold is raised high enough so that nodes are less sensitive to channel activities. Simulations are used to validate our conjecture and are presented in Section 4.

In addition, in our analysis and simulations, we also assume that a node communicates directly only with other nodes that are within its omni-directional transmission range $R$, and will not communicate directly with nodes outside $R$ and inside its directional transmission range $R^{\prime}$.

\subsection{Throughput}

The throughput $T h$ of each directional collision avoidance scheme can be calculated by the proportion of time that a node spends transmitting data packets successfully in the average. With some simplifications, all the directional collision avoidance schemes we address in this paper can be analyzed using the same node model of Fig. 1, and differ only in the duration of certain states and the transition probabilities among these states. Let $\pi_{s}, \pi_{w}$ and $\pi_{f}$ denote the steady-state probability of states succeed, wait and fail respectively. From the node model of Fig. 1 we have:

$$
T h=\frac{\pi_{s} \cdot l_{\text {data }}}{\pi_{w} T_{w}+\pi_{s} T_{s}+\pi_{f} T_{f}}
$$

where $T_{s}, T_{f}$ and $T_{w}$ are the duration of states succeed, fail and wait, respectively.

We first derive those steady-state probabilities, transition probabilities and times spent at different states that are common to DTOR and DTDR, and then derive the results that are particular to each scheme.

It is easy to shown [8] that for all schemes

$$
T_{s}=l_{r t s}+l_{c t s}+l_{d a t a}+l_{a c k}+4 \text { and } T_{w}=1 .
$$

Given that a node in the wait state listens omni-directionally, the transition probability $P_{w w}$ that node $x$ continues to stay in wait state in a slot equals the probability that it does not initiate any transmission and there is no node around it initiating a transmission in the direction towards node $x$. Because these two events are independent, we have that

$$
P_{w w}=(1-p) e^{-p^{\prime} N^{\prime}}
$$

where $p^{\prime}=p \theta /(2 \pi)$ and $N^{\prime}$ is the average number of nodes within the directional transmission range $\left(R^{\prime}\right)$ of a node. 
According to [8], we have

$$
\begin{aligned}
& \pi_{w}=\frac{1}{2-P_{w w}}=\frac{1}{2-(1-p) e^{-p^{\prime} N^{\prime}}} \\
& \pi_{s}=\pi_{w} P_{w s}=\frac{P_{w s}}{2-(1-p) e^{-p^{\prime} N^{\prime}}} \\
& \pi_{f}=1-\pi_{w}-\pi_{s} .
\end{aligned}
$$

To derive the transition probability $P_{w s}$ from wait to succeed, we need to calculate the probability $P_{w s}(r)$ that node $x$ successfully initiates a four-way handshake with node $y$ at a given time slot when the two nodes are at a distance $r$ apart. The configuration is shown in Fig. 2, where $\theta$ is the beamwidth of transmissions and receptions. In Fig. 2, solid circles indicate omni-directional transmission ranges of nodes, while dashed circles indicate directional transmission ranges.

The success of the handshake between nodes $x$ and $y$ depends on the nodes for which $y$ is within their omni-directional transmission range and those nodes for which $y$ is within their directional transmission range. Fig. 2 indicates in dashed lines the area around nodes $x$ and $y$ that may contain nodes whose directional transmissions can reach $x$ or $y$. To simplify our computation of throughput, we assume that there are, in effect, $N^{\prime}$ nodes around a node's omni-directional transmission range, though no node is assumed to communicate directly with any other node that is only reachable from directional transmissions. In fact, this simplifying assumption avoids the complexity of calculating interference directly from those nodes that are between the solid and dashed circles and instead such interference is taken into account by increasing the number of nodes within omni-directional range from $N$ to $N^{\prime}$.

From Fig. 2, we can see that the region around nodes $x$ and $y$ can be divided into five areas. The sizes of the five areas have been calculated in Ref. [8] and are omitted for brevity.

With the above definitions, $P_{w s}(r)$ equals the probability that $x$ transmits in a given time slot, $y$ does not transmit in the same time slot, and none of the nodes in the five areas defined above interfere with the handshake between $x$ and $y$. Given that transmissions are independent, we have:

$$
P_{w s}(r)=p_{x} \cdot p_{y} \cdot \prod_{i=1}^{5} p_{i}
$$

where

$$
\begin{aligned}
& p_{x}=\text { Prob. }\{x \text { transmits in the time slot }\}=p, \\
& p_{y}=\text { Prob. }\{y \text { does not transmit in the time slot }\}=1-p, \\
& p_{i}=\text { Prob. }\{\text { none of the nodes within Area } i \text { interfere } \\
& \quad \text { with the handshake between } x \text { and } y\} .
\end{aligned}
$$

The next two subsections compute the remaining periods and probabilities needed to compute the throughput of the DTOR and DTDR schemes. 


\subsection{The DTOR Scheme}

The DTOR scheme has been analyzed in Ref. [8] and we just cite the results here.

$$
\begin{aligned}
& T_{f}=\frac{1-p}{1-p^{T_{2}-T_{1}+1}} \sum_{i=0}^{T_{2}-T_{1}} p^{i}\left(T_{1}+i\right) \\
& p_{1}=e^{-p S_{1} N^{\prime}} \\
& p_{2}=e^{-p^{\prime} S_{2} N^{\prime}\left(2 l_{r t s}\right)} \cdot e^{-p S_{2} N^{\prime}} \\
& p_{3}=e^{-p^{\prime} S_{3} N^{\prime}\left(2 l_{r t s}+l_{c t s}+l_{\text {data }}+l_{a c k}+4\right)} \\
& p_{4}=e^{-p^{\prime} S_{4} N^{\prime}\left(2 l_{r t s}+l_{c t s}+l_{a c k}+2\right)} \\
& p_{5}=e^{-p^{\prime} S_{5} N^{\prime}\left(3 l_{r t s}+l_{\text {data }}+2\right)} \\
& P_{w s}=\int_{0}^{1} 2 r\left(p \cdot(1-p) \cdot p_{1} \cdot p_{2} \cdot p_{3} \cdot p_{4} \cdot p_{5}\right) d r
\end{aligned}
$$

Please note that we need to substitute $N^{\prime}$ for $N$ when considering the possible higher gain in directional transmissions.

\subsection{The DTDR Scheme}

Referring to Fig. 2, it is clear that, for the DTDR scheme, only nodes in Areas 1 and 2 can interfere with the handshake between nodes $x$ and $y$. However, in the DTDR scheme, nodes are more vulnerable to the transmissions from other nodes in these areas than they are in the DTOR scheme, because nodes receive omni-directionally only if they are in the wait state. To take the higher vulnerability into account, we use $l_{r t s}+l_{c t s}+2$ as the lower bound for the distribution of $T_{f}$ which still follows a truncated geometric distribution [8].

On the other hand, because nodes $x$ and $y$ are immune to the transmissions from nodes in Areas 3,4, and 5, and because concurrent transmissions can go on unobstructed in these areas, we introduce a spatial reuse factor $\gamma(r)$ for this scheme in the calculation of $P_{w s}(r)$. The parameter $\gamma(r)$ is defined to be the number of possible concurrent transmissions in the combined region covered by nodes $x$ and $y$, i.e., $\gamma(r)=\min \left(\gamma_{1}(r), \gamma_{2}(r)\right)$. In the expression, $\gamma_{1}(r)$ is the ratio between the total region covered nominally by nodes $x$ and $y$ and the actual region covered by the handshake between nodes $x$ and $y$. If there is one handshake in Areas 1 and 2, then in theory there can be possibly $\gamma_{1}(r)$ concurrent handshakes in the total area of Areas 1 to 5. Hence, $\gamma_{1}(r)=\left(S_{1}+S_{2}+S_{3}+S_{4}+\right.$ $\left.S_{5}\right) /\left(S_{1}+S_{2}\right)$. On the other hand, $\gamma_{2}(r)$ can be explained as follows: There are on average $N \cdot\left(S_{3}+S_{4}+S_{5}\right)$ nodes in the area of Areas 3 to 5 and in theory they can form a maximum of $\gamma_{2}(r)=N \cdot\left(S_{3}+S_{4}+S_{5}\right) / 2$ pairs of concurrent handshakes. To be conservative, we take the smaller value of $\gamma_{1}(r)$ and $\gamma_{2}(r)$ to estimate the spatial reuse benefit enabled by the $D T D R$ scheme.

It should be noted that the above is a very crude estimation of the gain in spatial reuse for the $D T D R$ scheme. Still, for this scheme, $P_{w s}$ can be adjusted as follows:

$$
P_{w s}=\int_{0}^{1} 2 r \gamma(r) P_{w s}(r) d r
$$


The other quantities needed to derive the throughput are the same as those for the DTOR scheme.

\subsection{Analytical Results}

We compare the performance of the OTOR, DTOR and DTDR schemes. For the OTOR scheme, we use the results reported by Wang and Garcia-Luna-Aceves [8], who assume that correct collision avoidance is enforced.

We present the results of two typical configurations for the three schemes; similar results can be readily obtained for other configurations. In these configurations, $\tau$ denotes the duration of one slot and RTS, CTS, and ACK packets last $5 \tau$, while a data packet lasts $100 \tau$. In configuration one, both omni-directional and directional transmissions have the same gain and thus we have $N^{\prime}=N$. In configuration two, directional transmissions have higher gain than omni-directional transmissions and $\gamma=1.5$. In this case, $N^{\prime}=$ $1.5^{2} \mathrm{~N}=2.25 \mathrm{~N}$.

For each configuration, we derive the maximum achievable throughput when the antenna beamwidth changes from $\theta=15^{\circ}\left(\frac{\pi}{12}\right)$ to $\theta=120^{\circ}(2 \pi / 3)$ in increment of $\theta=15^{\circ}\left(\frac{\pi}{12}\right)$. The results for configurations one and two are shown in Figs. 3 and 4 , respectively.

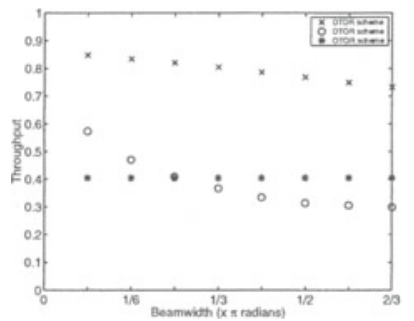

(a) Throughput $(\mathrm{N}=3)$

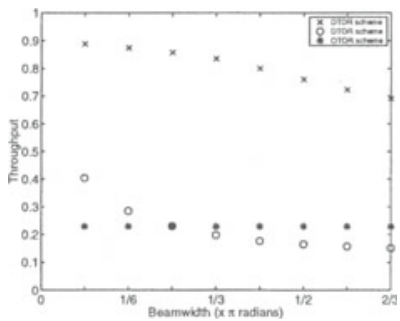

(b) Throughput $(\mathrm{N}=8)$

Fig. 3. Throughput comparison when omni-directional and directional transmissions have equal gain $\left(l_{r t s}=l_{c t s}=l_{a c k}=5 \tau, l_{\text {data }}=100 \tau, \gamma=1\right)$.

Figs. 3 and 4 clearly show that the DTDR scheme maintains the highest throughput among the three schemes, even with the increase of transmission and receiving beamwidth. Two factors contribute to the superiority of the DTDR scheme. One is the significant increase in spatial reuse, because only a small area is covered by the transmissions between two nodes engaged in a handshake according to the analysis. The other is the much reduced interference from those nodes that are not aware of the handshake because of directional receiving.

Even though the DTDR scheme does not ensure perfect collision avoidance, the directional reception capability makes the receiving node immune to the transmissions from many other nodes in Areas 3, 4, and 5 after it transmits a CTS packet. Hence, in 
terms of avoiding collisions, the DTDR scheme is as good as or even better than the $O T O R$ scheme, which silences all the neighbors around both a sender and a receiver.

Another significant advantage of the DTDR scheme is that its performance does not degrade with the increase of competing nodes within a neighborhood when antenna beamwidth is narrow. Instead, it even has a slight increase in throughput. This can be explained as follows: When the number of nodes is small, spatial reuse may be not utilized to its full advantage because some nodes may have to stay idle when all of their neighbors are engaged. This is not due to collision avoidance, but due to the scarity of nodes. Hence, when more nodes are around, the effect of spatial reuse is more conspicuous and one-hop throughput increases accordingly. However, when antenna beamwidth increases, spatial reuse is reduced and throughput still degrades with the increase of $N$ as people usually expect.

The results in Figs. 3 and 4 also show that, as expected, the performance of the $D T O R$ and DTDR schemes degrades when directional transmissions have higher gain than omni-directional transmissions. This is a direct consequence of the fact that the higher gain of directional transmissions leads to more interference at nodes receiving in omni-directional mode. However, it is clear that the DTDR scheme is superior to the $O T O R$ scheme in all cases, and the same conclusion can be derived from the results of simulation experiments described in Section 4. This helps to justify our approach of having each node consider as its neighbors those nodes that it hears through their omni-directional beacon transmissions.

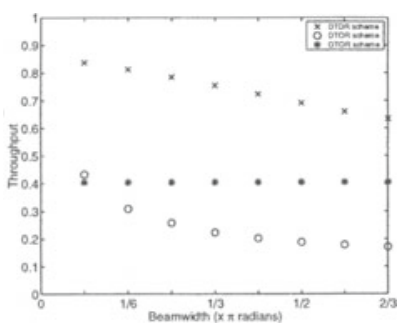

(a) Throughput $(\mathrm{N}=3)$

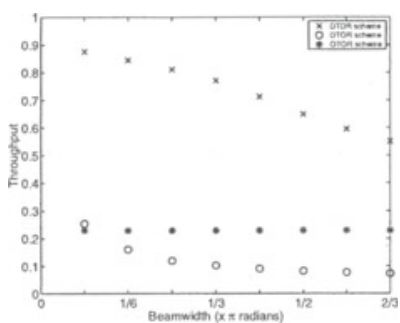

(b) Throughput $(\mathrm{N}=8)$

Fig. 4. Throughput comparison when the gain of directional transmissions equals one and a half times the gain of omni-directional transmissions $\left(l_{r t s}=l_{c t s}=l_{a c k}=5 \tau, l_{\text {data }}=100 \tau, \gamma=\right.$ 1.5).

\section{Simulation Results}

This section describes the results of computer simulations used to investigate the performance of the popular IEEE 802.11 DFWMAC protocol, which is labeled as OTOR in this section, and its variants corresponding to three directional collision avoidance schemes. The directional schemes considered are the DTOR, MTDR and DTDR schemes. 
We use GloMoSim 2.0 as the network simulator and implement the directional collision avoidance schemes under the assumption that there is a neighbor protocol that maintains a list of neighbors as well as their locations by means of beacons transmitted omni-directionally and periodically.

In addition to evaluating the performance of the directional collision avoidance schemes with ideal directional antennas, we have also evaluated the performance of these schemes with directional antennas that generate side lobes in directional transmissions.

\subsection{Performance Evaluation with Ideal Directional Antennas}

Direct sequence spread spectrum (DSSS) parameters are used throughout the simulations, which are shown in Table 1 . The raw channel bit rate is $2 \mathrm{Mbps}$. We use a uniform distribution to approximate the Poisson distribution used in our network model. In this network model, we place nodes in concentric circles or rings and focus on the performance of the innermost $N$ nodes in networks with radius of $3 R$. The network model has been described in detail in [8] and is omitted here for brevity.

Table 1. IEEE 802.11 protocol configuration parameters

\begin{tabular}{|c|c|c|c|c|c|c|}
\hline RTS & CTS & data & ACK & DIl & & SI \\
\hline 20-byte & 14-byte & 1460-byte & 14-byte & $50 \mu \mathrm{s}$ & & $10 \mu \mathrm{sec}$ \\
\hline contenti & in winc & slot time & e sync. & $\operatorname{me} \mid \mathrm{F}$ & & \\
\hline & $31-10$ & $20 \mu \mathrm{sec}$ & $192 \mu$ & usec & & \\
\hline
\end{tabular}

In our simulation, each node has a constant-bit-rate (CBR) traffic generator with data packet size of 1460 bytes, and one of its neighbors is randomly chosen as the destination for each packet generated. All nodes are always backlogged. We run simulation programs with $N=3,5$, and 8 , and for each choice of $N$ we use beamwidth values of $\theta=30^{\circ}$, $90^{\circ}$, and $150^{\circ}$. The same beamwidth is used for directional transmissions and receptions for simplicity.

Fifty random topologies were generated that satisfy the uniform distribution and the average of the throughput for the $N$ nodes in the innermost circle of radius $R$ was computed for each configuration.

The results for the case in which omni-directional and directional transmissions have equal gain are shown in Fig. 5. The results for the case in which directional transmissions have higher gain than omni-directional transmissions and $\gamma=1.5$ are shown in Fig. 6.

In Figs. 5-6, the vertical lines show the range of throughput achieved by each scheme, i.e., mean \pm standard variance. The lines are shifted a bit for clarity. The DTDR scheme performs the best among all these schemes and its performance does not degrade even for large values of $N$ as predicted in the analysis when antenna beamwidth is narrow. The results also show that the MTDR scheme outperforms the DTOR scheme, which indicates that the directional receiving capability can boost performance significantly.

Without directional receiving, a scheme with mixed transmissions ( $M T$ scheme) performs worse than a scheme with only directional transmissions (DT scheme). This is 


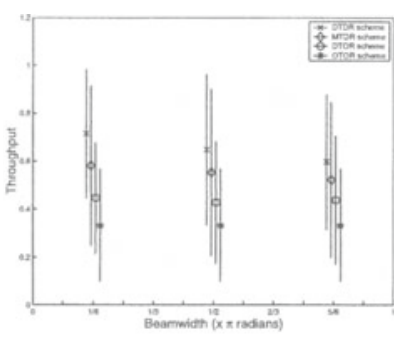

(a) $\mathrm{N}=3$

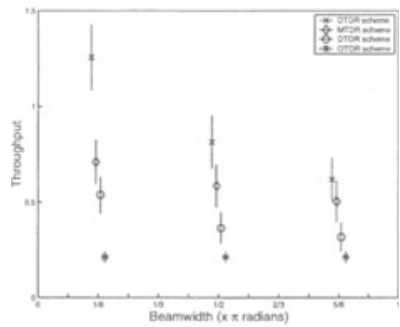

(b) $\mathrm{N}=8$

Fig. 5. Throughput comparison - equal gain

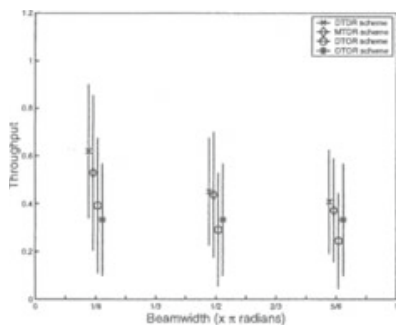

(a) $\mathrm{N}=3$

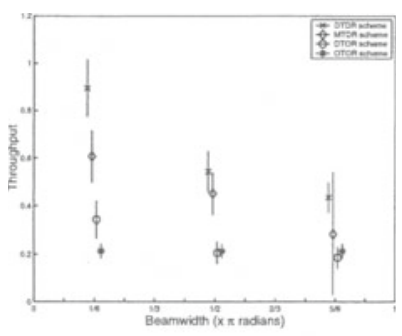

(b) $\mathrm{N}=8$

Fig. 6. Throughput comparison - higher gain $(\gamma=1.5)$

because omni-directionally transmitted CTS packets make almost all the nodes around the receiver defer their access to the shared channel or interfere with the ongoing handshake around the nodes that transmit CTS packets. Such conservative collision avoidance can largely nullify the benefits of spatial reuse and an all-directional scheme such as $D T$ is shown to perform much better than $M T$ when both schemes use only directional transmission capability of antenna systems.

However, when directional receiving is used, even though CTS is transmitted omnidirectionally, the handshakes of those nodes that have turned their receiving to other directions are not affected. Hence, the MTDR scheme can outperform the DTOR scheme in this case, although its performance is still inferior to the DTDR scheme because of the reduced spatial reuse.

It is also clear that, when beamwidth becomes wider, the performance of the DTDR scheme degrades faster when $N$ becomes larger. This shows that when networks are dense, the performance of a directional scheme is more influenced by the transmission/reception beamwidth.

It should be noted again that, because correct collision avoidance is not enforced in the IEEE 802.11 MAC protocol, collisions of data packets can still occur and hence the 
OTOR scheme cannot achieve the same performance predicted in the analysis, which assumes correct collision avoidance. It is for this reason that the DTOR scheme performs better than the OTOR scheme, even when wider beamwidths are used.

When comparing the results shown in Fig. 5 with those in Fig. 6, it is clear that higher directional transmission gains can have negative effects on both throughput and delay (which is not shown here due to limited space). This is because a node's directional transmissions interfere with more nodes, which translates into a reduction in spatial reuse, given that more nodes spend more time in the wait state after perceiving the channel busy.

We also find that the schemes with narrow receiving beamwidth have far smaller data packet collision ratios than the schemes without directional receiving. Hence, with directional receiving, the adverse effects of hidden terminals are almost completely canceled, leading to much higher throughput. It can also be noted that higher directional transmission gain leads to higher data collision ratio due to the increased interference it introduces. Therefore, directional antenna systems that are able to transmit and receive with narrow beamwidth while having the capability to reduce the power of directional transmissions are much more desirable than other variants.

\subsection{Impact of Side Lobes}

As discussed in Section 3.1, we conjecture that side lobes in directional transmissions should not have much effect on throughput if the side lobe level is low enough and carrier sensing threshold is raised. Hence, we implemented the directional antenna model in GloMoSim and conducted some simulations. In our simulations, we also set the interference range of side lobes $d=R / \gamma$. Other configurations remain the same as the case when directional transmissions have higher gain, i.e., $R^{\prime}=\gamma R$. Due to the limited space, we just summarize the results. We find that the presence of side lobes has negligible effect on throughput and it only causes larger variance for access delay when $N$ is small.

\section{Conclusion}

We have presented an analytical modeling of directional collision avoidance schemes that takes into account directional transmission and reception capabilities and the possibility of having different gains in omni-directional and directional transmissions. The analytical results show that the scheme in which all transmitting and receiving are done directionally can achieve much higher throughput than any other hybrid scheme that combines directional and omni-directional transmissions or receptions. Furthermore, the one-hop throughput of the all-directional scheme does not degrade with the increase of competing nodes within a region, which shows that the all-directional scheme is also much more scalable in dense ad hoc networks. It is also shown that higher directional transmission gain can have negative effects on the performance of directional collision avoidance schemes due to the increased interference range and reduced spatial reuse.

Extensive simulations of the popular IEEE 802.11 MAC protocol and its directional variants validate the analytical results. Simulation results also show that side lobes have 
little effect on throughput if side lobes are reasonably suppressed and carrier sensing threshold is raised to make nodes less sensitive to channel activities. Augmented with prior work that shows broadcast traffic does not degrade much the performance of directional collision avoidance schemes [12], it is argued that an all-directional scheme is very attractive and practical for ad hoc networks.

In practice, some form of power control to achieve similar gains for both omnidirectional and directional transmissions is desirable to take full advantage of the antenna systems. It is also possible to use power control to reduce both interference and energy consumption. Interesting areas of future research include analyzing the impact of eliminating omni-directional transmissions and receptions altogether by means of a directional beaconing mechanism, and comparing the performance of such a scheme against schemes that rely on omni-directional beaconing.

\section{References}

1. IEEE, IEEE Standard for Wireless LAN Medium Access Control (MAC) and Physical Layer (PHY) Specifications. IEEE Std 802.11-1997, IEEE, 1997.

2. Y. Wang and J. J. Garcia-Luna-Aceves, "Performance of Collision Avoidance Protocols in Single-Channel Ad Hoc Networks," in Proc. of IEEE ICNP '02, (Paris, France), Nov. 2002.

3. Y.-B. Ko, V. Shankarkumar, and N. H. Vaidya, "Medium Access Control Protocols Using Directional Antennas in Ad Hoc Networks," in IEEE INFOCOM 2000, Mar. 2000.

4. A. Nasipuri, S. Ye, J. You, and R. E. Hiromoto, "A MAC Protocol for Mobile Ad Hoc Networks Using Directional Antennas," in Proc. of the IEEE WCNC 2000, (Chicago, IL, USA), Sept. 2000.

5. R. Ramanathan, "On the Performance of Ad Hoc Networks with Beamforming Antennas," in ACM MobiHoc '01, (Long Beach, CA, U.S.A.), Oct. 2001.

6. M. Takai, J. Martin, A. Ren, and R. Bagrodia, "Directional Virtual Carrier Sensing for Directional Antennas in Mobile Ad Hoc Networks," in ACM MobiHoc '02, (Lausanne, Switzerland), June 2002.

7. R. R. Choudhury, X. Yang, R. Ramanathan, and N. Vaidya, "Medium Access Control in Ad Hoc Networks Using Directional Antennas,' in ACM MobiCom '02, (Atlanta, GA, USA), Sept. 2002.

8. Y. Wang and J. J. Garcia-Luna-Aceves, "Collision Avoidance in Single-Channel Ad Hoc Networks Using Directional Antennas," in Proc. of IEEE ICDCS '03, (Providence, RI, U.S.A.), May 2003.

9. H. Takagi and L. Kleinrock, "Optimal Transmission Range for Randomly Distributed Packet Radio Terminals," IEEE Trans. on Comm., vol. 32, no. 3, pp. 246-57, 1984.

10. F. Cali, M. Conti, and E. Gregori, "Dynamic Tuning of the IEEE 802.11 Protocol to Achieve a Theoretical Throughput Limit," IEEE/ACM Trans. on Net., vol. 8, pp. 785-799, Dec. 2000.

11. L. Wu and P. Varshney, "Performance Analysis of CSMA and BTMA Protocols in Multihop Networks (I). Single Channel Case," Information Sciences, Elsevier Sciences Inc., vol. 120, pp. 159-77, 1999.

12. Y. Wang and J. J. Garcia-Luna-Aceves, "Broadcast Traffic in Ad Hoc Networks with Directional Antennas," in IEEE Globecom 2003, (San Francisco, CA, U.S.A.), Dec. 2003. 